\title{
Role of dentate gyrus in aligning internal spatial map to external landmark
}

\author{
Jong Won Lee, ${ }^{1}$ Woon Ryoung Kim, ${ }^{2}$ Woong Sun, ${ }^{2}$ and Min Whan Jung ${ }^{1,3}$ \\ ${ }^{1}$ Neuroscience Laboratory, Institute for Medical Sciences, Ajou University School of Medicine, Suwon 443-721, Korea; \\ ${ }^{2}$ Department of Anatomy, College of Medicine, Korea University, Seoul 136-705, Korea
}

\begin{abstract}
Humans and animals form internal representations of external space based on their own body movement (dead reckoning) as well as external landmarks. It is poorly understood, however, how different types of information are integrated to form a unified representation of external space. To examine the role of dentate gyrus (DG) in this process, we conducted physiological and behavioral experiments using Bax knockout (Bax-KO) mice in which newly generated granule cells continue to accumulate disrupting neural circuitry specifically in the DG. Unlike in wild-type (WT) littermates, spatial firing of hippocampal neurons was completely dissociated from a distinct visual cue and instead, tended to stay constant relative to the recording room in Bax-KO mice. Behaviorally, whereas spatial learning was intact in conventional spatial reference memory tasks, Bax-KO mice were impaired in finding a target location based on visual landmarks when target locations predicted by dead reckoning and visual landmarks were made incongruent. These results provide converging evidence for the role of DG in binding animal's internal spatial map with the sensory information on external landmarks in building a distinct spatial representation for each environment.
\end{abstract}

[Supplemental material is available online at http://www.learnmem.org.]

Hippocampal neurons across different subregions show locationspecific discharges in rodents ("place cells") (O'Keefe and Dostrovsky 1971; Jung and McNaughton 1993), suggesting that allocentric spatial maps of external environments (cognitive maps) are represented in the hippocampus (O'Keefe and Nadel 1978). Discharge locations of place cells are controlled by external sensory cues, especially by distal visual cues, indicating that hippocampal spatial firing can be driven by external sensory information. On the other hand, place cell activity can be maintained in the absence of external sensory information, indicating that the information derived from the animal's own body movement is also sufficient to drive hippocampal spatial firing $\left(\mathrm{O}^{\prime}\right.$ Keefe and Nadel 1978; Redish 1999). These results indicate that hippocampal spatial representations are constructed from diverse sources of sensory information, which is consistent with a large body of behavioral studies demonstrating the capability of humans and animals in keeping track of their spatial positions based on idiothetic as well as allothetic cues (O'Keefe and Nadel 1978; Gallistel 1990; Redish 1999). It is currently unclear, however, how different types of sensory information are integrated to form a unified representation of external space.

In the present study, we focused on the potential role of the dentate gyrus (DG) in integrating incoming sensory information in constructing internal spatial representations, which is suggested by anatomical and physiological studies. Characteristics of grid cells in the medial entorhinal cortex (EC) (Hafting et al. 2005; Sargolini et al. 2006) suggest representation of internally generated (i.e., dead reckoning-based) spatial maps in this brain structure. By contrast, neurons in the lateral EC show little spatially selective firing (Hargreaves et al. 2005), suggesting the segregation of spatial and nonspatial information processing in the EC (Redish 1999; Knierim et al. 2006; Kerr et al. 2007). Because

${ }^{3}$ Corresponding author.

E-mail min@ajou.ac.kr; fax +82-31-219-4530.

Article is online at http://www.learnmem.org/cgi/doi/10.1101/lm.1483709. medial and lateral EC inputs converge in the DG and CA3, it has been proposed that internally generated spatial maps are associated with the sensory information on external landmarks in the DG-CA3 network, forming a distinct spatial context for each environment (Redish and Touretzky 1997; Hafting et al. 2005; O'Keefe and Burgess 2005; Knierim et al. 2006; Witter and Moser 2006; Gorchetchnikov and Grossberg 2007; Leutgeb and Leutgeb 2007). To test this hypothesis, we conducted physiological and behavioral experiments using Bax knockout (Bax-KO) mice, in which newly generated granule cells progressively accumulate disrupting neural circuitry (reductions in dendritic arborization of DG neurons and synaptic contact ratio of mossy fibers with CA3 dendritic spines), synaptic transmission (altered input-output relationship in mossy fiber-CA3 pyramidal cell synapses), and synaptic plasticity (reduced long-term potentiation in EC-granule cell and mossy fiber-CA3 pyramidal cell synapses) specifically in the DG and its output projections to CA3 (Supplemental Text Section 1; Supplemental Fig. S1; Sun et al. 2004; Kim et al. 2009). Bax-KO mice are different from DG-lesioned or DG-inactivated animals in that incoming sensory information is processed in the DG and transmitted to CA3. Bax-KO mice are also different from recently reported animal models lacking an $\mathrm{N}$-methyl-D-aspartate (NMDA) receptor subunit in the DG (McHugh et al. 2007; Niewoehner et al. 2007) in that not only synaptic plasticity but also neural circuitry and synaptic transmission are abnormal. In this respect, Bax-KO mice provide a unique opportunity to examine consequences of disrupted information processing in the DG. Exploiting these features, we compared hippocampal neuronal activity and spatial learning of Bax-KO mice with those of wild-type (WT) littermates (control).

\section{Results}

Characteristics of neuronal discharge

EEG and single unit signals were recorded from the DG and CA1 of 14 WT littermates and nine Bax-KO mice (6-8 mo old). Average 
running speed during the recording sessions was slightly higher in Bax-KO mice ( $n=33$ and 29 sets of three daily sessions for control and Bax-KO mice, respectively; two-way ANOVA, main effect of genotype, $F_{(1,60)}=9.345, P=0.003$; Fig. $\left.1 \mathrm{~A}\right)$, but this difference did not affect the subsequent analysis results; similar results were obtained after matching average running speeds of the two groups (data not shown). The distribution of relative $\theta$ EEG power was significantly different between the two genotypes in the DG: BaxKO mice had a stronger low frequency component $(6-9 \mathrm{~Hz})(n=13$ for both genotypes, three daily sessions were combined; $t$-test, $\left.t_{(24)}=-4.621, P<0.001\right)$, but a weak high frequency component $\left(9-12 \mathrm{~Hz}\right.$; $t$-test, $t_{(24)}=2.759, P=0.011$; Fig. 1B). No significant difference in the distribution of $\theta$ power was observed in CA1 $(n=$ 20 and 16 for control and Bax-KO mice, respectively; $t$-test, $6-9 \mathrm{~Hz}$ : $t_{(34)}=-0.635, P=0.530 ; 9-12 \mathrm{~Hz}, t_{(34)}=0.773, P=0.445$; Fig. $\left.1 \mathrm{~B}\right)$. The average firing rate of granule cells was also higher in Bax-KO mice $(n=34$ and 30 neurons for control and Bax-KO mice, respectively; two-way ANOVA, main effect of genotype, $F_{(1,62)}=$ 11.747, $P=0.001$; Fig. $1 \mathrm{C})$, but the average firing rate of CA1 neurons was similar between the two genotypes $(n=46$ and 51 neurons for control and Bax-KO mice, respectively; two-way ANOVA, main effect of genotype, $F_{(1,95)}=0.067, P=0.797$; Fig. 1C). These results indicate that abnormal accumulation of granule cells in Bax-KO mice induced global changes in basic physiological characteristics in the DG, but not in CA1.

Characteristics of spatial firing also differed between control and Bax-KO mice. Both CA1 complex spike cells and dentate granule cells recorded from Bax-KO mice tended to show less focused, scattered firing over the recording arena (Fig. 2A,D). This was reflected in low spatial information content per spike (twoway ANOVA, CA1: main effect of genotype, $F_{(1,95)}=53.808, P<$ 0.001; DG: main effect of genotype, $F_{(1,62)}=34.67, P<0.001$; Fig. 1D), low in-field/out-field firing ratio (only those neurons with "place fields" were analyzed; $t$-test, CA1, control: $n=33,28$, and 27, Bax-KO: $n=32,21$, and 31 for sessions 1,2 , and 3, respectively; $t_{(63)}=4.796, P<0.001 ; t_{(47)}=3.283, P=0.002 ;$ and $t_{(56)}=3.769, P<$ 0.001 for sessions 1,2 , and 3, respectively; DG, control: $n=28,19$, and 18, Bax-KO: $n=12,14$, and 11 for sessions 1,2 , and 3 , respectively; $t_{(38)}=2.036, P=0.049 ; t_{(31)}=2.904, P=0.007$; and $t_{(27)}=2.820, P=0.009$ for sessions 1,2 , and 3 , respectively; Fig. $\left.1 \mathrm{E}\right)$, and the tendency for smaller place field size (t-test, CA1: $t_{(63)}=$

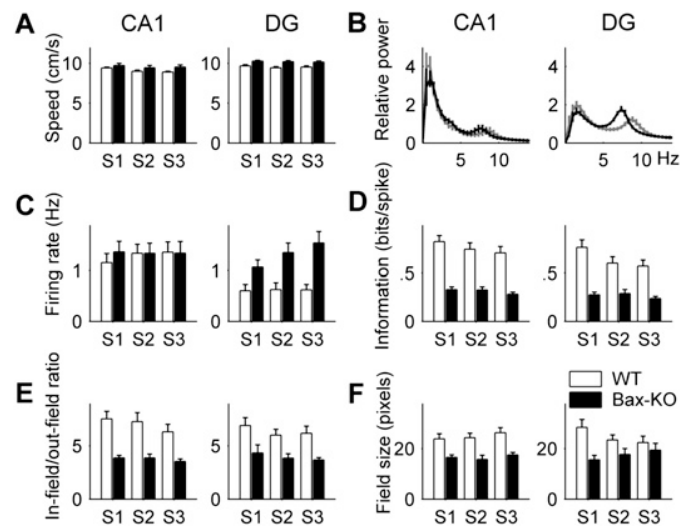

Figure 1. Characteristics of $C A 1$ pyramidal cell and granule cell discharge recorded from WT littermates and Bax-KO mice. $(A)$ Averaged running speed of the animals during $C A 1$ and DG recording sessions. ( $B$ ) Averaged EEG power spectrum. Bin width, $0.5 \mathrm{~Hz}$. Three daily sessions were combined. Gray indicates WT littermates; black, Bax-KO mice. (C) Mean discharge rate. $(D)$ Spatial information content per spike. $(E)$ Infield/out-field firing ratio. $(F)$ Average size of place fields. S1, S2, and S3 indicate sessions 1,2, and 3, respectively.
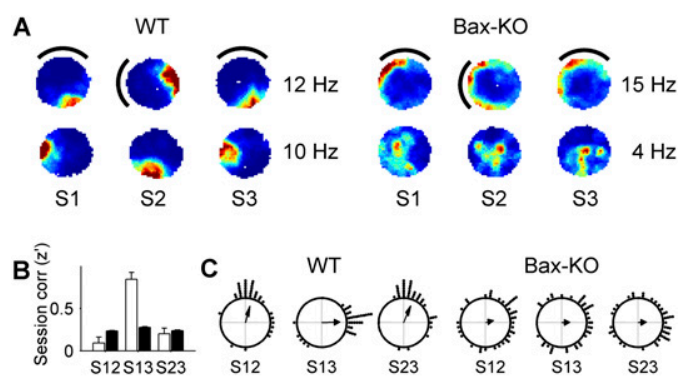

D
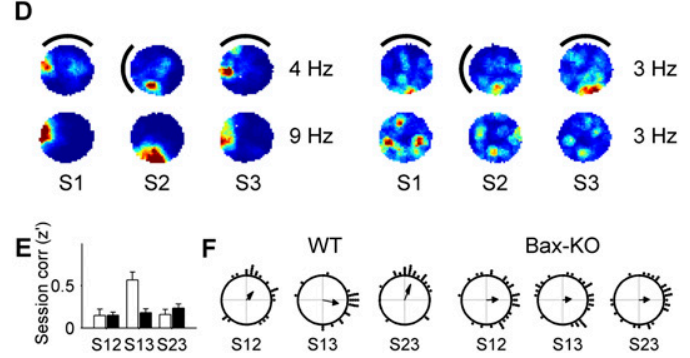

Figure 2. Dependence of spatial firing on external visual cue. $(A)$ Two examples of spatial firing rate maps for CA1 pyramidal cells recorded from WT littermates and Bax-KO mice. Red indicates maximum firing rate that is different for each cell (indicated on the right of session 3 spatial firing rate map). The visual cue (indicated by black arcs) was rotated $90^{\circ}$ counterclockwise in session 2. (B) Pixel-by-pixel correlation (z-transformed value) of spatial firing rate maps between sessions for CA1 pyramidal cells. S12, S13, and S23 indicate sessions 1 and 2, 1 and 3, and 2 and 3, respectively. (C) Distribution of rotation correlation scores of CA1 pyramidal cells. $(D-F)$ DG data. The formats are the same as in $A-C$.

$3.130, P=0.003 ; t_{(47)}=3.273, P=0.002 ;$ and $t_{(56)}=4.003, P<0.001$ for sessions 1,2 , and 3, respectively; DG: $t_{(38)}=2.568, P=0.014$; $t_{(31)}=1.820, P=0.078$; and $t_{(27)}=0.764, P=0.452$ for sessions 1,2 , and 3, respectively; Fig. 1F) in Bax-KO mice.

\section{Dependence of spatial firing on sensory cue}

Across sessions, CA1 and DG neurons in control animals emitted spikes relative to the visual cue, but those in Bax-KO mice showed activity that was independent on the location of the visual cue (Fig. 2A,D). In control animals, pixel-by-pixel correlation (converted to $Z$-scores for normalization) (Song et al. 2005) between spatial firing rate maps was high between sessions 1 and 3 (CA1: $n=37, z=0.84 \pm 0.08$, DG: $n=23, z=0.57 \pm 0.09$ ), in which the visual cue card was on the same location, but low between sessions 1 and 2 (CA1: $z=0.09 \pm 0.07$, DG: $z=0.15 \pm 0.08$ ) and sessions 2 and 3 (CA1: $z=0.20 \pm 0.07$, DG: $z=0.16 \pm 0.06$ ), in which the visual cue card was on different locations. In contrast, correlations were similarly low across all three comparisons in Bax-KO mice (39 CA1 and 28 DG neurons; CA1: $z=0.23 \pm 0.01$, DG: $z=0.15 \pm$ $0.08, \mathrm{CA} 1: z=0.28 \pm 0.01, \mathrm{DG}: z=0.18 \pm 0.04$ and CA1: $z=0.24 \pm$ 0.01 , DG: $z=0.23 \pm 0.05$ for sessions 1 vs. 2,1 vs. 3 , and 2 vs. 3 , respectively; Fig. 2B,E). The spatial correlation across sessions 1 and 3 was significantly different between the two genotypes (twoway ANOVA; CA1: main effect of genotypes, $F_{(1,74)}=2.958, P=$ 0.090 , main effect of session pairs, $F_{(2,148)}=49.216, P<0.001$, effect of genotype $\times$ session pair interaction, $F_{(2,148)}=38.895, P<$ 0.001 ; post-hoc Bonferroni comparisons across genotypes: sessions 1 and $2, P=0.110$, sessions 1 and $3, P<0.001$, sessions 2 and 3 , $P=0.655 ;$ DG: main effect of genotypes, $F_{(1,49)}=3.715, P=0.060$, main effect of session pairs, $F_{(2,98)}=8.864, P<0.001$, effect of genotype $\times$ session pair interaction, $F_{(2,98)}=9.676, P<0.001$; posthoc Bonferroni comparisons across genotypes: sessions 1 and $2, P=$ 0.968 , sessions 1 and $3, P<0.001$, sessions 2 and $3, P=0.323$ ). 
Rotation correlation scores (Knierim 2002; Song et al. 2005), which measure the rotation angle of the second firing rate map that produces the highest correlation score, were strongly clustered near $90^{\circ}$ between sessions 1 and 2 and between sessions 2 and 3 , but near $0^{\circ}$ between sessions 1 and 3 in control animals, whereas they were broadly distributed and only weakly clustered near $0^{\circ}$ across all sessions in Bax-KO mice (Fig. 2C,F). The distribution of rotation correlation scores was significantly different between the two genotypes (rank-sum test, sessions 1 and 2, CA1: $P<0.001$, DG: $P=0.027$; sessions 2 and 3, CA1: $P<0.001$, DG: $P=0.002$; sessions 1 and 3, CA1: $P<0.001$ ) except for the DG units across sessions 1 and 3 , which showed the same trend $(P=$ $0.052)$. In control animals, the direction of the mean vector was $75.55^{\circ}$ (CA1) and $51.62^{\circ}$ (DG) between sessions 1 and 2; 67.62 (CA1) and $69.44^{\circ}$ (DG) between sessions 2 and 3 ; and $1.63^{\circ}$ (CA1) and $347.16^{\circ}$ (DG) between sessions 1 and 3 . Substantial deviations of the mean vector from $90^{\circ}$ between sessions 1 and 2 and between sessions 2 and 3 were because of a small number of cells that maintained their spatial firing relative to the recording room ("partial remapping") (e.g., Knierim 2002). There were 27 (out of 37) CA1 and 11 (out of 23) DG neurons with the rotation correlation score within $90 \pm 30^{\circ}$ between sessions 1 and 2 , which were significantly above chance (binomial test, $P<0.001$ for both regions). Similarly, there were $26 \mathrm{CA} 1$ and $13 \mathrm{DG}$ neurons with the rotation correlation score within $90 \pm 30^{\circ}$ between sessions 2 and 3 , which were significantly above chance (binomial test, $P<0.001$ for both regions). Between sessions 1 and 3 , the rotation correlation score was significantly clustered near $0^{\circ}\left(n=31 \mathrm{CA} 1\right.$ and $15 \mathrm{DG}$ neurons within $0 \pm 30^{\circ}$, respectively; binomial test, $P<0.001$ for both regions). These results further demonstrate the dependence of neuronal discharge on the location of the visual cue in WT littermates.

Unlike in WT littermates, the direction of the mean vector was close to $0^{\circ}$ across all sessions in Bax-KO mice (CA1: $14.31^{\circ}, 3.54^{\circ}$, and $1.58^{\circ}$ for sessions 1 and 2,2 and 3 , and 1 and 3 , respectively; DG: $2.90^{\circ}, 2.94^{\circ}$, and $6.74^{\circ}$ for sessions 1 and 2,2 and 3 , and 1 and 3 , respectively; Fig. 2C,F). Although rotation correlation scores were broadly distributed, the length of the mean vector was significantly larger than 0 in all conditions (Rayleigh test; CA1: $P=0.010,0.010$, and 0.014 for sessions 1 and 2, 2 and 3, and 1 and 3, respectively; DG: $P=0.002,0.006$, and 0.033 for sessions 1 and 2,2 and 3 , and 1 and 3 , respectively). Across sessions 1 and 2, there were only five and four CA1 and DG neurons, respectively, with the rotation correlation score within $90 \pm 30^{\circ}$, which was not significantly above chance (binomial test; $P=0.802$ and 0.709 for CA1 and DG, respectively). On the other hand, the numbers of neurons with the rotation correlation score within $0 \pm 30^{\circ}(n=11$ CA1 and 11 DG neurons) were, albeit small, significant (binomial test, $P=0.050$ and 0.004 , respectively). Similarly, for the other session pairs, the rotation correlation score was significantly clustered near $0^{\circ}$ (sessions 2 and 3: $n=18 \mathrm{CA} 1$ and $13 \mathrm{DG}$ neurons within $0 \pm 30^{\circ}, P<$ 0.001 for both regions; sessions 1 and 3: $n=13$ CA1 and 10 DG neurons within $0 \pm 30^{\circ} ; P=0.008$ and 0.012 , respectively), but not near $90^{\circ}$ (sessions 2 and $3: n=6$ CA 1 and 4 DG neurons within $90 \pm 30^{\circ} ; P=0.651$ and $P=0.709$, respectively; sessions 1 and $3: n=5$ CA1 and 3 DG neurons within $90 \pm 30^{\circ} ; P=0.802$ and 0.868 , respectively) in Bax- $\mathrm{KO}$ animals. The different distributions of rotation correlation scores between Bax-KO mice and WT littermates were not because of different selectivity of spatial firing (Supplemental Text Section 2; Supplemental Fig. S2). These results show that hippocampal neurons in Bax-KO mice fired independent of the location of the visual cue and instead, tended to fire at the original location with respect to the recording room.

\section{Performance in water maze}

The above results predict impaired landmark-based navigation in Bax-KO mice. We therefore compared spatial learning of control and Bax-KO mice in a Morris water maze task. Both genotypes (seven Bax-KO mice and eight WT littermates, 6 mo old, batch 1) improved their performance similarly over the course of $6 \mathrm{~d}$ of training (three trials per day), as indexed by the latency to escape to a submerged platform (two-way ANOVA, main effect of training session: $F_{(5,65)}=18.142, P<0.001$, no significant effect of genotype: $F_{(1,13)}=0.578, P=0.461$; no significant interaction: $F_{(5,65)}=$ $0.185, P=0.967$; Fig. 3B). However, in the probe trial on the seventh day, in which the platform and distal visual cues were rotated $144^{\circ}$ clockwise with respect to the testing room and the entry point (Fig. 3A), the escape latency of Bax-KO mice was significantly longer than that of WT littermates ( $t$-test, $t_{(13)}=$ 4.569, $P=0.001$; Fig. 3B). Thus, Bax-KO mice relied less on the landmarks in finding the escape platform in spite of normal vision (Supplemental Text Section 3; Supplemental Fig. S3). When we measured the average distance of the animal from the original platform location (Gallagher et al. 1993; Maei et al. 2009) on day 7, it was significantly shorter for Bax-KO mice ( $t$-test; all trials, $t_{(40)}=$ $3.183, P=0.003$, first trial only, $t_{(12)}=2.698, P=0.019$; Fig. $\left.3 \mathrm{C}, \mathrm{D}\right)$, indicating that Bax-KO mice tended to navigate to the old goal location with respect to the testing room.

\section{Performance on radial maze}

Another batch of animals (five WT littermates and five Bax-KO mice, $8-10$ mo old, batch 2) was tested in a spatial reference memory task on a six-arm radial maze. Both genotypes improved their performance over the course of $8 \mathrm{~d}$ of training (four trials per day), as indexed by the number of errors (visiting nonrewarding arms, two-way ANOVA; main effect of training session, $F_{(7,56)}=$ 4.429, $P=0.001$; no significant effect of genotype, $F_{(1,8)}=0.017$, $P=0.899$; no significant interaction, $F_{(7,56)}=0.805, P=0.587$; Fig. $4 \mathrm{~A}$ ) and the latency to reach the goal location (two-way ANOVA; main effect of training session, $F_{(7,56)}=5.747, P<0.001$; no significant effect of genotype, $F_{(1,8)}=3.508, P=0.098$; no significant interaction, $F_{(7,56)}=1.699, P=0.128$; Fig. $\left.4 \mathrm{~B}\right)$, which is consistent with our previous finding (Kim et al. 2009). On the subsequent days (days 9 and 10), we rotated together the maze and the surrounding visual cues (also the target arm) trial-by-trial in a random manner in steps of $60^{\circ}$. Compared with control animals,

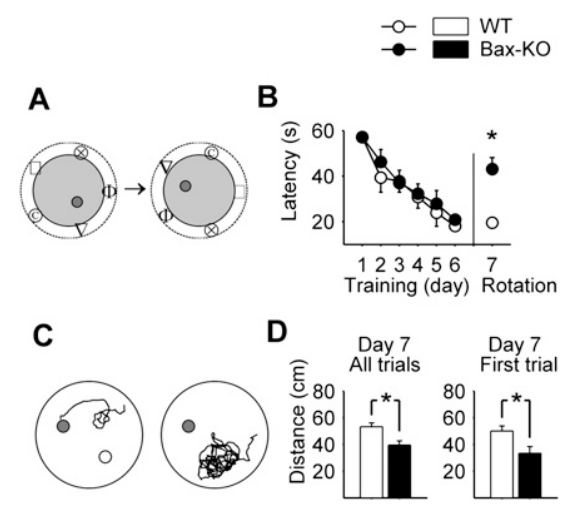

Figure 3. Behavioral performance in Morris water maze task. (A) A schematic diagram of the water maze and surrounding visual cues. On probe trial (day 7), visual cues and the platform location were rotated $144^{\circ}$ clockwise. $(B)$ The latency to find a hidden platform over $7 \mathrm{~d}$ of training. Each data point consists of three daily trials. (C) Example movement trajectory of a control (left) and a Bax-KO mouse (right) during a probe trial on day 7 . The white circle denotes the location of the original (but absent) platform. (D) The average distance of the animal from the center of the original platform on day 7 (sampling frequency, $10 \mathrm{~Hz}$ ). (Left) All trials were analyzed. (Right) Only the first trial was analyzed. $\left(^{*}\right)$ Significant difference. 

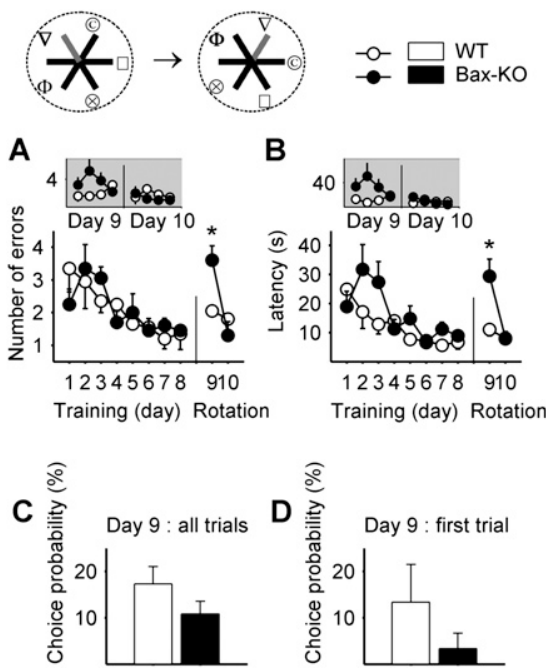

Figure 4. Behavioral performance in radial maze spatial reference memory task. Animals were trained to find water reward at the end of one arm. Behavioral performances in the course of $10 \mathrm{~d}$ of training are shown in terms of the number of errors $(A)$ and the latency to reach the goal location $(B)$. Following $8 \mathrm{~d}$ of training, the maze, the target location, and visual cues were rotated together randomly in steps of $60^{\circ}$, as indicated by the top diagram, for two additional days. Each data point consists of four daily trials. (Insets) Trial-by-trial performance of the animals during the last $2 \mathrm{~d}$ on which the maze was randomly rotated. $(C, D)$ The probability to choose the original goal arm relative to the testing room on day 9. (C) All trials were analyzed. (D) Only the first trial was analyzed. (*) Significant difference.

Bax-KO mice were severely impaired in this task on the ninth day (Fig. 4A,B).

Unlike in the water maze experiment, however, no significant tendency was observed for Bax-KO mice to choose the original goal arm relative to the testing room on the ninth day ( $t$-test; all trials, $t_{(38)}=1.389, P=0.173$; first trial, $t_{(8)}=1.134, P=$ 0.290; Fig. 4C,D). Also, on the next day (day 10), the performance of Bax-KO mice was similar to that of WT littermates (two-way ANOVA, number of errors: main effect of training session, $F_{(1,8)}=$ 12.626, $P=0.007$, significant effect of genotype $\times$ session interaction, $F_{(1,8)}=8.160, P=0.021$; post-hoc Bonferroni comparison across genotypes, day $9, P=0.010$, day $10, P=0.464$; latency: main effect of training session, $F_{(1,8)}=16.645, P=0.004$, significant effect of genotype $\times$ session interaction, $F_{(1,8)}=9.581, P=0.015$; post-hoc Bonferroni comparison across genotypes, day $9, P=$ 0.019 , day $10, P=0.952$; Fig. $4 \mathrm{~A}, \mathrm{~B})$. Examination of trial-by-trial performance following maze rotation revealed gradual improvement in the performance of Bax-KO mice, but not of control mice (linear regression analysis, number of errors; Bax-KO mice: $P<0.001$; WT littermates: $P=0.383$; latency, Bax-KO: $P<0.001$; WT littermates: $P=0.319$ ), indicating that new learning took place in Bax-KO mice but not in WT littermates (Fig. 4A,B, gray-shaded insets).

\section{Discussion}

\section{Role of DG in aligning internal spatial map to external landmark}

Distal visual cues in general exert strong control over spatial firing of hippocampal neurons. In WT littermates, as expected, discharge locations of the majority of CA1 and DG neurons were anchored to a distal visual cue. On the other hand, in Bax-KO mice, spatial firing of hippocampal neurons was completely dissociated from the visual cue, which was not attributable to impaired vision or low spatial selectivity of hippocampal neuronal activity. Behaviorally, upon the rotation of a maze, Bax-KO mice were impaired in finding a target location based on visual cues. However, when needed, Bax-KO mice were capable of landmarkbased spatial learning on the radial maze, suggesting that the original deficit is not because of a failure to represent external landmarks but because of a failure to use this information for spatial navigation. These results provide converging evidence that the DG is involved in binding animal's internal spatial representation with the sensory information on external landmarks. It would be important for future studies to examine how this function of the DG is related to other proposed functions of the DG. For example, our results are compatible with the "pattern separation" role of the DG (for review, see Kesner 2007; Treves et al. 2008), because formation of environment-specific spatial maps will allow fine discrimination of spatial locations where important events took place.

Not only DG, but also CA3, has been proposed to play a role in binding internal spatial maps with the sensory information on external landmarks (Redish 1999; Hargreaves et al. 2005; Knierim et al. 2006; Kerr et al. 2007). However, we failed to find any structural or functional abnormality in the direct EC projection pathway to CA3 in Bax-KO mice (Kim et al. 2009). Although we cannot rule out the possibility that abnormal firing characteristics of DG granule cells significantly affected CA3 neuronal activity in Bax-KO mice, our results suggest that the direct EC-CA3 pathway alone is insufficient to support internal spatial map-external landmark association. On the other hand, mice lacking an NMDA receptor subunit specifically in CA3 showed well-maintained positional firing of CA1 neurons when they were returned to the original recording arena, and these animals showed intact spatial learning in a Morris water maze task but were impaired under a partial cue condition (Nakazawa et al. 2002), suggesting that they employed map-based navigation with the reference framework bound to external landmarks. Thus, whereas the contribution of CA3 to this process cannot be excluded, our results suggest that intact DG is required in binding together internal spatial maps and external landmarks. The anatomy and physiology of the DG are also consistent with the possibility. The synapses formed by direct EC projections comprise the major population for granule cells ( $\sim 80 \%$ ) (Amaral et al. 1990; Amaral and Witter 1995), suggesting strong influences of direct EC inputs on granule cell discharge. In addition, the threshold for the activation of granule cells is relatively high (Lomo 1971), raising the possibility that granule cells are activated only when sufficiently strong inputs from both the medial and lateral EC converge.

Interestingly, when needed, Bax-KO mice were capable of landmark-based spatial learning. It is possible that landmark-based spatial learning was mediated by the direct EC-CA1 projection system in Bax-KO mice. This pathway has been shown to be able to support incremental spatial learning (Nakashiba et al. 2008). Alternatively, the EC-CA3 projection pathway or disrupted DG of Bax-KO mice might be able to support landmark-based spatial learning with extended training. Finally, we cannot rule out the possibility that Bax-KO mice, upon a failure to find a goal location based on dead reckoning, switched their behavioral strategy so as to simply approach toward a visual cue that was near the target arm rather than to employ map-based navigation (taxon vs. locale navigation) (O'Keefe and Nadel 1978; Redish and Touretzky 1997), which can be supported by the basal ganglia (Packard and Knowlton 2002).

Internal spatial representation based on dead reckoning What was the navigation strategy employed by Bax-KO mice and how was spatial firing constructed in Bax-KO mice without relying on external landmarks? Considering a large body of evidence 
indicating the capability of humans and animals in building allocentric spatial representations based on their own body movement (O'Keefe and Nadel 1978; Gallistel 1990; Redish 1999; Etienne and Jeffery 2004), one obvious candidate is dead reckoning. This is also supported by the characteristics of grid cells found in the medial EC that suggest the representation of dead reckoning-based spatial maps in this brain structure (Hafting et al. 2005; Sargolini et al. 2006). Our results are in general consistent with this possibility. Spatial firing in Bax-KO mice tended to stay constant relative to the recording room, and Bax-KO mice tended to visit the original platform location in the water maze upon the rotation of visual cue(s). Although this tendency was not observed in the radial maze task, this might be due to the fact that the degree of freedom of movement was too low to reveal the animal's navigation strategy. Spatial firing/navigation in reference to the testing room allows only two explanations: the use of dead reckoning strategy or unknown room-bound sensory cues. We can exclude the use of local cues in the physiological experiment because the arena itself was rotated and the floor paper was replaced between sessions. It is also unlikely that Bax-KO mice used room-bound olfactory or auditory cues in the recording arena or in the water maze because (1) we controlled potential roombound cues in a careful manner, (2) Bax-KO animals were indifferent from WT animals in auditory and olfactory sensitivity (Supplemental Text Section 3; Supplemental Fig. S3), and (3) it is difficult to imagine that Bax-KO mice relied on other faint (if such existed) sensory cues ignoring distinct visual cues in spite of normal vision (Supplemental Text Section 3; Supplemental Fig. S3). Then, unless we assume that Bax-KO mice used an unknown earthbound sense of direction, which a large body of behavioral studies failed to find, it would be reasonable to conclude that BaxKO mice relied on dead reckoning in navigating toward the hidden platform and maintaining spatial firing of hippocampal neurons.

A potential problem for the argument that dead reckoning was behind spatial firing of hippocampal neurons as well as spatial navigation of Bax-KO mice is the difference in spatial precision. Whereas Bax-KO mice were as good as control animals in finding a target location when dead reckoning was allowed, spatial firing of hippocampal neurons in Bax-KO mice was poorly correlated across sessions. Although the reason for this difference is currently unclear, it might be because of different experimental procedures between the physiological and behavioral experiments. Most importantly, animals were not required to keep track of their positions in the physiological experiments, because there was no specific goal to find. Also, the entry point into the maze was precisely fixed in the behavioral experiments, whereas it was more variable in the physiological experiments. Finally, unit recording persisted longer (10 min) than the animal's goal-searching behavior (typically $<1 \mathrm{~min}$ ), which might have caused drifting of place fields in Bax-KO mice due to cumulative dead reckoning errors (Gallistel 1990; Etienne and Jeffery 2004). Spatial firing of hippocampal neurons in Bax-KO mice might be more strongly bound to the recording room if these factors are controlled.

\section{Strong influence of trisynaptic pathway on discharges of CAl neurons}

Inconsistent results have been reported on the relative importance of the direct EC-CA1 projection vs. the trisynaptic circuit pathway in driving spatially selective firing of CA1 neurons (Brun et al. 2002, 2008; Nakashiba et al. 2008; Suh et al. 2008). Our results show that abnormal features of granule cell spatial firing (low spatial selectivity and independence on visual cue) are faithfully reflected in spatial firing of CA1 neurons in Bax-KO mice despite intact synaptic transmission and long-term synaptic plasticity in
EC-CA3 and CA3-CA1 projection pathways (Kim et al. 2009). It might be argued that the trisynaptic circuit pathway exerts abnormally strong influences on CA1 neuronal activity in adult Bax-KO mice, because they have many more granule cells than WT littermates. This is unlikely, however, because synaptic connectivity is adjusted in adult Bax-KO mice so that the total number of mossy fiber synapses onto CA3 neurons remains similar to that in WT littermates (Buss et al. 2006; Kim et al. 2009). Moreover, a previous study has shown that mice with selective loss of an NMDA receptor subunit in the DG have broadly tuned CA3 place cells (McHugh et al. 2007). Our results therefore suggest that the trisynaptic circuit pathway exerts stronger influence on spatial firing of CA1 neurons compared with the direct EC layer III projection to CA1, with the caveat that we have yet to examine synaptic transmission/plasticity of the direct EC-CA1 projection in Bax-KO mice. This is consistent with a much larger number of CA3-CA1 synapses compared with EC-CA1 synapses (Amaral et al. 1990), and the fact that the perforant path input to CA1 forms distal synapses so that it is hard to drive CA1 neuronal discharges without CA3-CA1 input activity (Jarsky et al. 2005). However, in vitro physiological and modeling studies have suggested nonlinear interactions between the perforant path and trisynaptic circuit pathway in activating CA1 neurons (Remondes and Schuman 2002; Jarsky et al. 2005). Thus, it remains to be determined how exactly the trisynaptic circuit pathway works together with the perforant path input in determining CA1 neuronal activity in vivo.

\section{Materials and Methods}

\section{Animals}

Bax-KO mice were maintained on a C57BL/6 background. Homozygous Bax-deficient (Bax-KO) and WT littermate mice were generated from matings between heterozygous males and females. Sibling animals were collected individually and genotyped by PCR (Knudson et al. 1995). Only those animals at 6-10 mo of age were used in the present study because DG circuit disruption becomes clear in Bax-KO mice at 6 mo of age (Sun et al. 2004; Kim et al. 2009). Separate groups of animals were used in behavioral (12 Bax$\mathrm{KO}$ mice and 13 WT littermates) and physiological (nine Bax-KO mice and 14 WT littermates) experiments. All experiments were carried out in accordance with the regulations and approval of the Ethics Review Committee for Animal Experimentation of Ajou University School of Medicine.

\section{Behavior}

\section{Morris water maze task}

The water maze (diameter, $1.55 \mathrm{~m}$ ) containing a hidden platform (diameter, $20 \mathrm{~cm}) 20 \mathrm{~cm}$ away from the wall, was filled with opaque water. The maze was surrounded by a black circular curtain (diameter, $2 \mathrm{~m}$ ) that contained five distinct visual cues (Fig. 3A). The animals were hand-carried from a designated location in the testing room to the maze without any disorientation, and the entry point through the curtain stayed constant relative to the testing room throughout the experiment. This procedure was to allow the animals to solve the task based on visual cues as well as dead reckoning. However, they were released at three different locations (three quadrants where the escape platform was not located) in random order so that the hidden platform could not be found based on a simple response strategy. The animals were allowed to find the hidden platform for $1 \mathrm{~min}$. If the animals could not find the platform within $1 \mathrm{~min}$, they were guided to the platform and remained there for $1 \mathrm{~min}$. Animals were trained three times a day for $6 \mathrm{~d}$. On the seventh day (probe trial), the curtain (and also the visual cues) and the location of the hidden platform were rotated $144^{\circ}$ clockwise and the animal's performance was tested. 


\section{Spatial reference memory task on radial maze}

The six-arm radial maze consisted of a central platform (diameter, $15 \mathrm{~cm}$ ) and six arms (length, $35 \mathrm{~cm}$; width, $5 \mathrm{~cm}$; height of transparent walls, $20 \mathrm{~cm}$ ). The maze was surrounded by a white curtain that contained five distinct visual cues (Fig. 4). The animals were water-deprived and motivated to find the water reward (volume, $20 \mu \mathrm{L}$ ) at the end of one (and always the same) arm. They were allowed to search for the target location until they found it. As in the water maze task, the animals were hand-carried from a designated location in the testing room to the maze without any disorientation, and the entry point through the curtain stayed constant relative to the testing room throughout the experiment. The animals were introduced to the center of the maze and confined there for $30 \mathrm{sec}$ surrounded by a 20 -cm-high black cylinder (diameter, $14 \mathrm{~cm}$ ) that blocked the animal's view of the visual cues before each trial began. Following $8 \mathrm{~d}$ of training (four trials per day), the maze, the surrounding curtain (and visual cues), and the target arm were rotated randomly in steps of $60^{\circ}$ in each trial to test whether animals relied on visual cues in finding the target location.

\section{Neurophysiology}

Four tetrodes were chronically implanted in the left or right dorsal hippocampus (1.7 $\mathrm{mm}$ posterior and $1.5 \mathrm{~mm}$ lateral to bregma) of mice (14 WT littermates and nine Bax-KO mice, 6-8 mo old) to record single unit activity from CA1 (nine WT littermates and seven Bax-KO mice) and DG (seven WT littermates and three BaxKO mice; both CA1 and DG neurons were recorded sequentially from two WT littermates and one Bax-KO mouse). In each recording session, animals were allowed to freely explore a circular arena (diameter, $30 \mathrm{~cm}$; height, $40 \mathrm{~cm}$ ) made of a black wall with one quarter of the wall covered with a white board that served as a visual cue. Unit signals were recorded in the following sequence: session 1 (10 min), intersession interval (10 min); session 2 (10 min, the arena was rotated $90^{\circ}$ counterclockwise), intersession interval (10 $\mathrm{min})$; session 3 (10 $\mathrm{min}$, the arena was rotated back to its original configuration). Fresh paper was placed on the floor before each recording session to avoid potential influence of odor cues across sessions. Unit signals were also recorded for $\sim 5 \mathrm{~min}$ on a pedestal outside the circular arena both before and after the main recording sessions to ensure the stability of recorded unit signals. Unit signals were amplified 10,000×, filtered between 600 and $6000 \mathrm{~Hz}$, digitized at $32 \mathrm{kHz}$, and stored on a personal computer using the Cheetah data acquisition system. EEG was recorded through one channel of each tetrode while recording unit signals from the cell body layer. EEG signals were amplified 500-1000×, filtered at $1-475 \mathrm{~Hz}$, and digitized at $1 \mathrm{kHz}$. Skull screws were used as the reference electrode for both unit and EEG recordings. The animal's head position was also monitored by tracking a lightemitting diode mounted on the headstage at $60 \mathrm{~Hz}$. When recordings were completed, small marking lesions were made by passing an electrolytic current $(30 \mu \mathrm{A}$, cathodal, $10 \mathrm{sec})$, and recording locations were verified histologically as previously described (Song et al. 2005).

\section{Analysis}

Relative $\theta$ power was quantified by performing fast Fourier transforms on EEG data and calculating the relative power of 6-9 (low frequency component) and 9-12 $\mathrm{Hz}$ (high frequency component) $\theta$ bands. Unit signals were isolated off-line based on various spike waveform parameters using the MClust software (AD Redish; Supplemental Fig. S4). Those units recorded in CA1 were classified into complex spike cells (mean firing rate, $<5 \mathrm{~Hz}$; spike width, $>250 \mu \mathrm{sec}$; and occasional bursts) and $\theta$ cells (the rest), and those recorded in the DG were classified into putative granule cells (mean firing rate, $<3.5 \mathrm{~Hz}$; spike width, $>250 \mu \mathrm{sec}$; and occasional bursts) and putative interneurons (the rest). Only complex spike cells (CA1) (Ranck Jr 1973) and putative granule cells (DG) (Jung and McNaughton 1993) with a mean firing rate $>0.1 \mathrm{~Hz}$ in at least one of three recording sessions were included in the analyses (CA1: $n=46$ and 51 for control and Bax-KO mice, respectively; DG: $n=34$ and 30 for control and Bax-KO mice, respectively).
When we compared spatial firing between sessions (i.e., computing spatial firing rate map correlation and rotation correlation score), only complex spike cells and putative granule cells with a mean firing rate $>0.1 \mathrm{~Hz}$ in all three recording sessions were included in the analyses (CA1: $n=37$ and 39 for control and BaxKO mice, respectively; DG: $n=23$ and 28 for control and Bax-KO mice, respectively). Spatial firing rate maps were constructed by dividing the $85.0 \times 63.7 \mathrm{~cm}$ rectangle containing the circular arena into $80 \times 60$ pixels, and calculating the firing rate for each pixel based on an "adaptive binning" procedure (Jung et al. 1994). A place field was defined as a group of 10 or more adjoining pixels with the average firing rate of each pixel exceeding three standard deviations above the mean firing rate. Pixel-by-pixel correlations between firing rate maps were computed and then transformed to Fisher's $z$ for normalization as previously described (Song et al. 2005). The rotation correlation score (Knierim 2002; Song et al. 2005) was quantified by calculating pixel-by-pixel correlation of firing rate maps between two sessions while rotating the firing rate map of the second session by $0^{\circ} \sim 350^{\circ}$ at $10^{\circ}$ intervals. The rotation angle that produced the highest correlation was taken as the rotation correlation score. Spatial information content per spike was calculated as previously described (Skaggs et al. 1993).

\section{Statistical analysis}

Student's $t$-test (two-tailed) and two-way ANOVA (repeated measure) were used for statistical comparisons. Rayleigh test, a ranksum test, and a binomial test were employed to examine statistical significance of rotation correlation scores (Batschelet 1981). A $P$-value $<0.05$ was used as the criterion for a significant statistical difference. All data are expressed as mean \pm SEM.

\section{Acknowledgments}

We thank A.D. Redish and L.M. Frank for helpful comments on the initial manuscript. This work was supported by Korea Research Foundation Grant KRF-2008-314-H00006, Korea Science and Engineering Foundation Grant 2005-000-10199-0, the 21st Century Frontier Research Program, and the Cognitive Neuroscience Program of the Korea Ministry of Science and Technology (M.W.J.).

\section{References}

Amaral DG, Witter MP. 1995. Hippocampal formation. In The rat nervous system. (ed. G Paxinos), pp. 443-493. Academic Press, San Diego, CA.

Amaral DG, Ishizuka N, Claiborne B. 1990. Neurons, numbers and the hippocampal network. Prog Brain Res 83: 1-11.

Batschelet E. 1981. Circular statistics in biology. Academic Press, London, UK.

Brun VH, Otnass MK, Molden S, Steffenach HA, Witter MP, Moser MB, Moser EI. 2002. Place cells and place recognition maintained by direct entorhinal-hippocampal circuitry. Science 296: 2243-2246.

Brun VH, Leutgeb S, Wu HQ, Schwarcz R, Witter MP, Moser EI, Moser MB. 2008. Impaired spatial representation in CA1 after lesion of direct input from entorhinal cortex. Neuron 57: 290-302.

Buss RR, Sun W, Oppenheim RW. 2006. Adaptive roles of programmed cell death during nervous system development. Annu Rev Neurosci 29: 1-35.

Etienne AS, Jeffery KJ. 2004. Path integration in mammals. Hippocampus 14: 180-192.

Gallagher M, Burwell R, Burchinal M. 1993. Severity of spatial learning impairment in aging: Development of a learning index for performance in the Morris water maze. Behav Neurosci 107: 618-626.

Gallistel CR. 1990. The organization of learning. MIT Press, Cambridge, MA.

Gorchetchnikov A, Grossberg S. 2007. Space, time and learning in the hippocampus: How fine spatial and temporal scales are expanded into population codes for behavioral control. Neural Netw 20: 182-193.

Hafting T, Fyhn M, Molden S, Moser MB, Moser EI. 2005. Microstructure of a spatial map in the entorhinal cortex. Nature 436: 801-806.

Hargreaves EL, Rao G, Lee I, Knierim JJ. 2005. Major dissociation between medial and lateral entorhinal input to dorsal hippocampus. Science 308: 1792-1794.

Jarsky T, Roxin A, Kath WL, Spruston N. 2005. Conditional dendritic spike propagation following distal synaptic activation of hippocampal CA1 pyramidal neurons. Nat Neurosci 8: 1667-1676.

Jung MW, McNaughton BL. 1993. Spatial selectivity of unit activity in the hippocampal granular layer. Hippocampus 3: 165-182. 
Jung MW, Wiener SI, McNaughton BL. 1994. Comparison of spatial firing characteristics of units in dorsal and ventral hippocampus of the rat. Neurosci 14: 7347-7356.

Kerr KM, Agster KL, Furtak SC, Burwell RD. 2007. Functional neuroanatomy of the parahippocampal region: The lateral and medial entorhinal areas. Hippocampus 17: 697-708.

Kesner RP. 2007. A behavioral analysis of dentate gyrus function. Prog Brain Res 163: 567-576.

Kim WR, Park OH, Choi S, Choi SY, Park SK, Lee KJ, Rhyu IJ, Kim H, Lee YK, Kim HT, et al. 2009. The maintenance of specific aspects of neuronal function and behavior is dependent on programmed cell death of adult-generated neurons in the dentate gyrus. Eur J Neurosci 29: 14081421.

Knierim JJ. 2002. Dynamic interactions between local surface cues, distal landmarks, and intrinsic circuitry in hippocampal place cells. J Neurosci 22: 6254-6264.

Knierim JJ, Lee I, Hargreaves EL. 2006. Hippocampal place cells: Parallel input streams, subregional processing, and implications for episodic memory. Hippocampus 16: 755-764.

Knudson CM, Tung KS, Tourtellotte WG, Brown GA, Korsmeyer SJ. 1995. Bax-deficient mice with lymphoid hyperplasia and male germ cell death. Science 270: 96-99.

Leutgeb S, Leutgeb JK. 2007. Pattern separation, pattern completion, and new neuronal codes within a continuous CA3 map. Learn Mem 14: 745757.

Lomo T. 1971. Patterns of activation in a monosynaptic cortical pathway: The perforant path input to the dentate area of the hippocampal formation. Exp Brain Res 12: 18-45.

Maei HR, Zaslavsky K, Teixeira CM, Frankland PW. 2009. What is the most sensitive measure of water maze probe test performance? Front Integr Neurosci 3: 4. doi: 10.3389/neuro.07.004.2009.

McHugh TJ, Jones MW, Quinn JJ, Balthasar N, Coppari R, Elmquist JK Lowell BB, Fanselow MS, Wilson MA, Tonegawa S. 2007. Dentate gyrus NMDA receptors mediate rapid pattern separation in the hippocampal network. Science 317: 94-99.

Nakashiba T, Young JZ, McHugh TJ, Buhl DL, Tonegawa S. 2008. Transgenic inhibition of synaptic transmission reveals role of CA3 output in hippocampal learning. Science 319: 1260-1264.

Nakazawa K, Quirk MC, Chitwood RA, Watanabe M, Yeckel MF, Sun LD, Kato A, Carr CA, Johnston D, Wilson MA, et al. 2002. Requirement for hippocampal CA3 NMDA receptors in associative memory recall. Science 297: 211-218.

Niewoehner B, Single FN, Hvalby Ø, Jensen V, Meyer zum Alten Borgloh S, Seeburg PH, Rawlins JNP, Sprengel R, Bannerman DM. 2007. Impaired spatial working memory but spared spatial reference memory following functional loss of NMDA receptors in the dentate gyrus. Eur J Neurosci 25: $837-846$.

O'Keefe J, Burgess N. 2005. Dual phase and rate coding in hippocampal place cells: Theoretical significance and relationship to entorhinal grid cells. Hippocampus 15: 853-866.

O'Keefe J, Dostrovsky J. 1971. The hippocampus as a spatial map. Preliminary evidence from unit activity in the freely-moving rat. Brain Res 34: 171-175.

O'Keefe J, Nadel L. 1978. The hippocampus as a cognitive map. Clarendon Press, Oxford, UK.

Packard MG, Knowlton BJ. 2002. Learning and memory functions of the basal ganglia. Annu Rev Neurosci 25: 563-593.

Ranck JB Jr. 1973. Studies on single neurons in dorsal hippocampal formation and septum in unrestrained rats. I. Behavioral correlates and firing repertoires. Exp Neurol 41: 461-531.

Redish AD. 1999. Beyond the cognitive map: From place cells to episodic memory. MIT Press, Cambridge, MA.

Redish AD, Touretzky DS. 1997. Cognitive maps beyond the hippocampus. Hippocampus 7: 15-35.

Remondes M, Schuman EM. 2002. Direct cortical input modulates plasticity and spiking in CA1 pyramidal neurons. Nature 416: 736-740.

Sargolini F, Fyhn M, Hafting T, McNaughton BL, Witter MP, Moser MB, Moser EI. 2006. Conjunctive representation of position, direction, and velocity in entorhinal cortex. Science 312: 758-762.

Skaggs WE, McNaughton BL, Gothard KM, Markus EJ. 1993. An information-theoretic approach to deciphering the hippocampal code. In Advances in neural information processing systems (eds. SJ Hanson et al.), pp. 1030-1037. Morgan Kaufmann, San Mateo, CA.

Song EY, Kim YB, Kim YH, Jung MW. 2005. Role of active movement in place-specific firing of hippocampal neurons. Hippocampus 15: 8-17.

Suh J, Rivest AJ, Tonegawa S. 2008. Genetic inducible blockade of the temporoammonic pathway: Spatial coding and acquisition of spatial and contextual information in the absence of direct cortical input. In Molecular and Cellular Cognition Society/Society for Neuroscience Satellite Symposium, p. SS57. Washington, DC.

Sun W, Winseck A, Vinsant S, Park OH, Kim H, Oppenheim RW. 2004. Programmed cell death of adult-generated hippocampal neurons is mediated by the proapoptotic gene Bax. J Neurosci 24: 11205-11213.

Treves A, Tashiro A, Witter MP, Moser EI. 2008. What is the mammalian dentate gyrus good for? Neuroscience 154: 1155-1172.

Witter MP, Moser EI. 2006. Spatial representation and the architecture of the entorhinal cortex. Trends Neurosci 29: 671-678.

Received May 13, 2009; accepted in revised form June 23, 2009. 


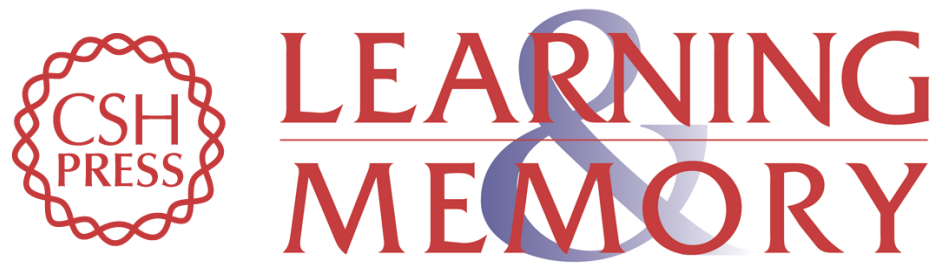

\section{Role of dentate gyrus in aligning internal spatial map to external landmark}

Jong Won Lee, Woon Ryoung Kim, Woong Sun, et al.

Learn. Mem. 2009, 16:

Access the most recent version at doi:10.1101//m.1483709

Supplemental http://learnmem.cshlp.org/content/suppl/2009/09/03/16.9.530.DC1
Material

References This article cites 36 articles, 11 of which can be accessed free at: http://learnmem.cshlp.org/content/16/9/530.full.html\#ref-list-1

License

Email Alerting Receive free email alerts when new articles cite this article - sign up in the box at the Service top right corner of the article or click here. 